\title{
Fas-Mediated Apoptosis of Melanoma Cells and Infiltrating Lymphocytes in Human Malignant Melanomas
}

Tetsuo Shukuwa, M.D., Ichiro Katayama, M.D., Takehiko Koji, Ph.D.

Departments of Dermatology (TS, IK) and Histology and Cell Biology (TK), Nagasaki University School of

Medicine, Nagasaki, Japan

In a rodent system, melanoma cells expressing Fas ligand (FasL) could kill Fas-positive lymphocytes, suggesting that FasL expression was an essential factor for melanoma cell survival in vivo. These findings led us to investigate apoptosis, and to histochemically analyze involvement of Fas and FasL in the induction of apoptosis, in human malignant melanoma tissues. The percentages of terminal deoxynucleotidyl transferase-mediated biotin-dUTP nick end-labeling (TUNEL)-positive melanoma cells and of proliferating cell nuclear antigen (PCNA)positive melanoma cells in melanoma tissues $(n=$ 22) were greater than those in melanocytes in uninvolved skin $(n=6)$ and nevus cells in nevi tissues $(n=9)$. The infiltrating lymphocytes around melanomas were also TUNEL positive. Immunohistochemistry revealed expression of Fas and FasL in melanoma cells and lymphocytes, whereas no Fas or FasL expression was detected in normal skin melanocytes and nevus cells. There was significant correlation between Fas-positive indices and TUNEL indices in melanoma tissues. Moreover, TUNEL-, Fas-, and FasL-positive indices of melanoma cells from patients with Stage 3 melanomas were significantly lower than those with Stage 2 melanomas. The PCNA index of Stage 1 melanoma was significantly lower than that of the other stages, although the difference of PCNA index was insignificant among Stages 2 to 4. Among Stages 1 to 4, there was no difference in the PCNA, TUNEL-, and Faspositive indices of lymphocytes, although the FasLpositive index of lymphocytes from Stage 3 melanomas was significantly lower than in that from Stage 2. These data reveal that melanoma cells and infil-

Copyright (C) 2002 by The United States and Canadian Academy of Pathology, Inc.

VOL. 15, NO. 4, P. 387, 2002 Printed in the U.S.A.

Date of acceptance: December 20, 2001.

This research was supported in part by a Grant-in-Aid (9770642) from the Ministry of Education, Science, Sports and Culture, Japan.

Address reprint requests to: Tetsuo Shukuwa, M.D., Department of Dermatology, Nagasaki University School of Medicine, 1-7-1 Sakamoto, Nagasaki 852-8501, Japan; fax: +81-95-849-7335; e-mail: shukuw-t@qa2.so-net.ne.jp. trating lymphocytes have the potential to induce their own apoptosis regulated by Fas and FasL in an autocrine and/or paracrine fashion and that the decline of Fas-mediated apoptosis of melanoma cells, rather than the apoptosis of infiltrating lymphocytes, may affect the prognosis of melanoma patients, possibly through the accumulation of more aberrant cells acquiring metastatic activity.

KEY WORDS: Apoptosis, Lymphocytes, Malignant melanoma, Melanocytic nevus, Proliferation.

Mod Pathol 2002;15(4):387-396

Tumor growth depends on the degree of imbalance between cell proliferation and cell loss (1). Cell loss is mostly due to cell death, which can be categorized into necrosis and apoptosis. In recent years, the biological importance of apoptosis has been recognized in tissue homeostasis (2-4), neurological disorders (5), autoimmune diseases (6), and various malignancies (7-11). Of particular interest has been the important role played by apoptosis in the development of cancers, including hepatocellular carcinoma (7), breast carcinoma (8), gastric cancer (9), colon cancer (10), and brain tumors (11). Among skin cancers, malignant melanoma is still one of the most aggressive malignant neoplasms and carries a poor prognosis (12). Mooney et al. (13) reported that the ratio of apoptotic to mitotic tumor cells in malignant melanomas is histologically lower than that in basal cell carcinomas. Moreover, Saida et al. (14) reported that the proliferation rate of tumor cells in malignant melanomas was lower than that of squamous cell carcinoma, although the prognosis of patients with melanomas is poorer than that of squamous cell carcinomas. Therefore, the frequency of apoptosis, rather than the proliferative activity in melanoma cells, may be an essential parameter that regulates the growth of melanomas.

Apoptosis is an active process of cell death, initially defined by Kerr et al. (15) on the basis of 
morphological features, which included shrinkage of the nucleus and cytoplasm, chromatin condensation to the nuclear periphery, and fragmentation of the cell. Histochemical detection by terminal deoxynucleotidyl transferase-mediated biotindUTP nick end-labeling (TUNEL) of doublestranded DNA breaks has been widely used as a simple method for identifying apoptotic nuclei (16). Significant attention is currently being paid to the process of apoptosis because it is induced by activation of specific genes, implying that cell death can be manipulated.

Fas (APO-1/CD95), an $M_{r} 45,000$ transmembranous glycoprotein, is a member of the tumor necrosis factor/nerve growth factor receptor family, known to be expressed in a variety of lymphoid and tumor cells (2, 3 , 17, 18). Fas ligand (FasL), a natural ligand of Fas, has recently been identified as an $M_{r} 31,000$ Type II transmembranous glycoprotein that initiates the apoptotic process in activated T lymphocytes by binding to surface Fas (18-20). Recently, Hahne et al. (21) reported that melanoma cells express FasL in metastatic lesions of human melanomas. Thus, melanoma cells seem to be capable of killing infiltrating Fasbearing activated lymphocytes adjacent to melanoma cells. Maeda et al. (22) also found that human melanoma cells expressed FasL in primary lesions, whereas there was no expression of FasL in melanocytic nevus cells and normal melanocytes. More recently, it has been reported that FasL-positive melanoma cells and TUNEL-positive lymphocytes were found in primary lesions of human melanomas (23, 24). It is possible that melanoma cells may induce apoptosis of lymphocytes mediated by Fas system. Furthermore, the expression of FasL on melanoma cells in metastatic lesions was correlated to the stages of melanomas (24). However, our knowledge of Fas expression in melanoma cells and of the interaction between melanoma cells and infiltratinglymphocytes in primary human malignant melanomas is still limited.

In this study, we focused on apoptosis of melanoma cells and infiltrating lymphocytes in human melanoma tissues. To gain insight into the mechanism of induction of apoptosis, we investigated the expression of Fas and FasL in melanoma tissues using immunohistochemical methods. To clarify the influence of apoptosis of melanoma cells and lymphocytes on the prognosis of melanoma patients, we also examined the correlation between frequency of apoptosis and stage of disease.

\section{MATERIALS AND METHODS}

\section{Reagents}

3,3' Diaminobenzidine-4 $\mathrm{HCl}$ (3,3'-diaminobenzidine) was purchased from Dojin Chemical Co.
(Kumamoto, Japan). Bovine serum albumin and Brij 35 were obtained from Sigma Chemical Co. (St. Louis, MO). The Apoptosis In Situ Detection Kit for TUNEL and all other reagents in this study were obtained from Wako Pure Chemical Industries (Osaka, Japan).

\section{Antibodies}

The antibodies used were polyclonal rabbit antiFas (Fas/APO-1/CD95) antibody (anti-Fas C; 2), polyclonal rabbit anti-FasL antibody (anti-P5; 2), and monoclonal mouse anti-proliferating cell nuclear antigen (PCNA) antibody (DAKO, Glostrup, Denmark, clone PC-10). The preparation and specificity of anti-Fas C (25) and anti-P5 (26) have been described elsewhere.

\section{Specimens of Malignant Melanoma}

Surgical specimens of cutaneous malignant melanoma lesions were obtained from 9 men and 13 women who ranged in age from 24 to 89 years. Table 1 lists the tumors studied, tumor classification, Breslow tumor thickness, Clark level of invasion (27), and tumor stage. Tumors were categorized into four stages based on the American Joint Cancer Council staging protocol: Stage 1, primary tumor of $0-1.5 \mathrm{~mm}$ thickness and no further spread; Stage 2, primary tumor of $1.5-4.0 \mathrm{~mm}$ thickness and no spread; Stage 3, primary lesions with tumor thickness of $>4.0 \mathrm{~mm}$ (Clark Level V) or local lymph node involvement; Stage 4 , spread beyond the local regional lymph nodes (28). The 22 melanoma specimens included four cases with Stage 1, five with Stage 2, six with Stage 3, and seven with Stage 4 melanomas. The patients with Stage 4 disease had distant metastases. No chemotherapeutic agents or biological response modifiers had been administered before surgery. As a control, surgical specimens of melanocytic nevi were obtained from five men and four women who ranged in age from 12 to 86 years. All patients gave informed consent for the clinical and laboratory research before commencement of the study.

\section{Tissue Preparation and Histopathology}

Surgical specimens were fixed in $10 \%$ neutralized formalin and embedded in paraffin according to a standard protocol. Serial sections $(4 \mu \mathrm{m}$ in thickness) were cut from the specimens and used for routine histological examination, TUNEL assay, and immunohistochemical analysis. These sections were mounted on aminopropyltriethoxysilanecoated slides (Matsunami Glass, Inc., Osaka, Japan). The paraffin sections were deparaffinized with $100 \%$ toluene at room temperature and thereafter hydrated stepwise through 100\%, 95\%, 90\%, 


\begin{tabular}{|c|c|c|c|c|c|c|}
\hline & Age & Sex & Histopathological Classification & Clark Level & Tumor Thickness (mm) & Stage \\
\hline 1 & 32 & Male & Acral lentiginous melanoma (ALM) & I & In situ & 1 \\
\hline 2 & 58 & Male & ALM & I & In situ & 1 \\
\hline 3 & 24 & Female & Superficisal spreading melanoma (SSM) & II & 0.5 & 1 \\
\hline 4 & 57 & Female & ALM & II & 0.6 & 1 \\
\hline 5 & 81 & Female & ALM & III & 1.3 & 2 \\
\hline 6 & 38 & Female & SSM & III & 1.6 & 2 \\
\hline 7 & 82 & Female & ALM & III & 1.7 & 2 \\
\hline 8 & 67 & Male & ALM & IV & 2.3 & 3 \\
\hline 9 & 54 & Female & ALM & IV & 2.7 & 2 \\
\hline 10 & 55 & Male & SSM & IV & 2.8 & 4 \\
\hline 11 & 87 & Female & ALM & IV & 2.9 & 3 \\
\hline 12 & 54 & Female & ALM & IV & 3.2 & 2 \\
\hline 13 & 50 & Female & Nodular melanoma (NM) & IV & 3.2 & 3 \\
\hline 14 & 69 & Male & ALM & IV & 3.5 & 4 \\
\hline 15 & 61 & Female & NM & IV & 3.7 & 4 \\
\hline 16 & 57 & Male & ALM & $\mathrm{V}$ & 5.0 & 3 \\
\hline 17 & 86 & Female & NM & $\mathrm{V}$ & 5.5 & 3 \\
\hline 18 & 68 & Male & ALM & V & 5.8 & 3 \\
\hline 19 & 60 & Male & NM & V & 6.0 & 4 \\
\hline 20 & 70 & Male & NM & V & 6.2 & 4 \\
\hline 21 & 45 & Female & NM & V & 6.8 & 4 \\
\hline 22 & 89 & Female & ALM & $\mathrm{V}$ & 8.0 & 4 \\
\hline
\end{tabular}

$80 \%$, and $70 \%$ ethanol baths. For histopathological examination, sections were stained with hematoxylin and eosin. After measurement of tumor thickness, melanoma specimens were classified as Clark Level I to V. To identify melanocytes, melanoma cells, and infiltrating lymphocytes, serially prepared hematoxylin and eosin-stained sections were used.

\section{Demelanization}

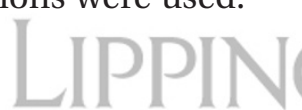

The majority of melanoma cells contained melanin granules, which often disturbed the observation of histochemical signals. To decolorize melanin in the samples, we optimized the conditions of demelanization in preliminary trials with $0.3 \%$ potassium permanganate and $0.2 \%$ oxalic acid. Optimum demelanization was obtained with successive use of $0.3 \%$ potassium mangan tetroxide for 4 minutes and $0.2 \%$ oxalic acid for 5 minutes.

\section{TUNEL Staining}

TUNEL assays were performed to identify nuclei with DNA strand breaks in histological sections, according to the instructions provided by the manufacturer. Briefly, deparaffinized sections were treated with proteinase $\mathrm{K}(5 \mu \mathrm{g} / \mathrm{mL})$ for 15 minutes at $37^{\circ} \mathrm{C}$, preincubated with terminal deoxynucleotidyl transferase buffer for 60 minutes, and then incubated with biotinylated 16-dUTP for $120 \mathrm{~min}$ utes at room temperature. After the sections were washed in phosphate-buffered saline (PBS), endogenous peroxidase activity was inactivated by immersing them in $3 \% \mathrm{H}_{2} \mathrm{O}_{2}$ in methanol for 5 minutes. The sections were then incubated with horseradish peroxidase (HRP)-labeled anti-biotin antibody (1:100) diluted with $5 \%$ bovine serum al-
bumin/PBS for 30 minutes at room temperature. After washing in PBS, the HRP-labeled sites were visualized with a chromogen solution, containing 3,3'-diaminobenzidine, $\mathrm{H}_{2} \mathrm{O}_{2}, \mathrm{CoCl}_{2}$, and $\mathrm{NiSO}_{4}\left(\mathrm{NH}_{4}\right)_{2} \mathrm{SO}_{4}$, to enhance signal intensity (29). After visualization, the sections were counterstained with $2 \%$ methyl green for 5 minutes. As a negative control, some slides were incubated without terminal deoxynucleotidyl transferase.

\section{Inmunohistochemistry \\ Fas and FasL}

Immunohistochemistry for Fas and FasL was performed according to the method reported previously by Wang et al. (3) with a slight modification, as described below. After demelanization, sections were immersed in $0.3 \% \mathrm{H}_{2} \mathrm{O}_{2}$ in methanol for 30 minutes and then preincubated with $500 \mu \mathrm{g} / \mathrm{mL}$ normal goat IgG in 1\% bovine serum albumin in PBS for 60 minutes at room temperature. They were then incubated with anti-Fas antibody (1:200) or anti-FasL antibody (1:200) diluted with $1 \%$ bovine serum albumin in PBS overnight at room temperature. After washing in PBS with $0.075 \%$ Brij 35 , a nonionic detergent, the slides were incubated with HRP goat anti-rabbit IgG (1:100). HRP-labeled sites were visualized using $\mathrm{H}_{2} \mathrm{O}_{2}$ and 3,3'-diaminobenzidine in $0.05 \mathrm{~m}$ Tris/HCl buffer $(\mathrm{pH}$ 7.6) for 10 minutes, and the slides were then counterstained with $2 \%$ methyl green for 5 minutes. As a control, some specimens were incubated with normal rabbit serum (1:200) in place of the primary antibody.

\section{Proliferating Cell Nuclear Antigen}

Immunostaining was performed according to the instructions provided with the DAKO Enhanced 
Polymer One-Step Staining PCNA antibody kit. Some sections were also incubated with DAKO Enhanced Polymer One-Step Staining Negative Control, containing rabbit and mouse immunoglobulins and HRP coupled to an inner polymer backbone without antibody. HRP-labeled sites were visualized by $\mathrm{H}_{2} \mathrm{O}_{2}$ and 3,3'-diaminobenzidine, and the slides were then counterstained with $2 \%$ methyl green for 5 minutes.

\section{Quantitative and Statistical Analysis}

The percentages of cells staining positive for the various antigens assayed were calculated in melanoma cells and infiltrating lymphocytes in melanomas, nevus cells in melanocytic nevi, and melanocytes in uninvolved skin tissues. The numbers of PCNA- and TUNEL-positive cells were counted in five fields under a light microscope at $200 \times$ magnification. To avoid a possible bias in counting, we selected five fields randomly to count a total of 2000-5000 per specimen, irrespective of the periphery part or center part of the tumor. In the case of the early-stage melanomas, we prepared consecutive sections and counted cells on every five sections to reach $\geq 2000$ cells of total count. The ratio of the number of TUNEL-positive cells to the number of PCNA-positive cells was calculated and expressed as the TUNEL-PCNA ratio. The ratio of the number of PCNA-positive cells to the total number of cells was determined as the PCNA index. TUNEL-, Fas- and FasL-positive indices were similarly calculated for each case. These results were expressed as a percentage (mean \pm SEM). To compare these indices among melanomas, melanocytic nevi and uninvolved skin, we performed the MannWhitney $U$ test. To elucidate the induction of apoptosis by expression of Fas and FasL, the correlations among TUNEL, Fas, and FasE indices of melanoma cells and lymphocytes were statistically analyzed using Spearman's rank correlation test. Mann-Whitney's $U$ test and Spearman's rank correlation test were performed using StatView software, Version 4.5 (Abacus Software, Inc., Berkeley, CA). To investigate the correlation between these indices and prognosis, these indices were also calculated for each stage of melanoma, and the statistical analysis was performed using Mann-Whitney's $U$ test. $P<.05$ was considered statistically significant.

\section{RESULTS}

Proliferative Activity Assessed by PCNA Staining in Melanoma Tissues

For the purposes of this study, it was essential to distinguish melanoma cells from lymphocytes.
Generally, lymphocytes contain scant cytoplasm and intensely stained small nuclei. On the basis of these histological features, identification of lymphocytes in hematoxylin and eosin-stained sections was possible. As shown in Figures 1A and 2A, PCNA-positive melanoma cells were abundant, whereas only a few positive lymphocytes were observed, and these were restricted to the periphery of the melanomas. In comparison, among melanoma cells, nevus cells, and melanocytes, the PCNA indices in melanocytic nevi and uninvolved skin were quite low (Fig. 3A). When the PCNA index of melanoma cells was compared among different stages, the index of Stage 1 melanoma was significantly lower than that of the other stages, and the difference in PCNA index was not statistically significant among Stages 2 to 4, as shown in Table 2. There was no statistically significant correlation between the PCNA index of lymphocytes and any melanoma stage (Table 3).

\section{Frequency of Apoptosis Assessed by}

\section{TUNEL Staining}

To identify cells with DNA strand breaks at an individual cell level, we performed TUNEL staining. In melanoma specimens, TUNEL-positive melanoma cells and lymphocytes with shrunken nuclei were randomly located (Figs. 1B, 2B). As shown in Figure 2B, the TUNEL index of melanoma cells $(0.64 \pm 0.06 \%)$ was significantly greater than that of nevus cells in melanocytic nevi $(0.40 \pm 0.05 \%$; $P<$ .05) and that of melanocytes in uninvolved skin (0.23 $\pm 0.03 \% ; P<$.05). Interestingly, TUNELpositive lymphocytes were found surrounding melanoma cells, and the TUNEL index of lymphocytes $(3.82 \pm 0.58 \%)$ was significantly higher than that of melanoma cells. Comparison between melanoma stages showed that the TUNEL indices of melanoma cells of Stages 3 and 4 were lower than that of cells of Stage 2, as shown in Table 2. In addition, when terminal deoxynucleotidyl transferase was omitted from the TUNEL reaction mixture as a negative control, the sections were essentially blank (data not shown).

\section{TUNEL/PCNA Ratio of Melanoma Cells}

The TUNEL-PCNA ratio was calculated to assess the balance between cell proliferation and cell death. As shown in Figure 3C, the ratio in melanoma cells was significantly lower than that in nevus cells $(P<.001)$ and that in melanocytes $(P<$ .0001). When the ratio for melanoma cells was compared among different stages of melanoma patients, an inverse correlation became evident, as shown in Table 2. In contrast, there was no significant correlation between the TUNEL-PCNA ratio 
in lymphocytes and melanoma stage (data not shown).

\section{Expression of Fas and FasL in Malignant Melanoma}

To assess the involvement of Fas and FasL in the induction of apoptosis in melanoma cells and infiltrating lymphocytes, we investigated Fas and FasL expression in melanoma tissues using immunohistochemistry. As shown in Figures $1 \mathrm{C}$ and 2C, some melanoma cells and lymphocytes were clearly Fas positive. FasL-positive melanoma cells and lymphocytes were also detected in the serial sections (Figs. 1D, 2D). However, we could not find the coincidence of Fas-positive cells and TUNELpositive cells in serial sections (data not shown). As shown in Figure 3D-E, the Fas index of melanoma cells was $0.31 \pm 0.03 \%$, and the FasL index was 0.33 $\pm 0.04 \%$. Nevus cells in melanocytic nevi and melanocytes in uninvolved skin were essentially negative for Fas and FasL.

The Fas indices of melanoma cells in Stage 3 and 4 melanoma cases were significantly lower than those of Stage 1 and $2(P<.05$, each, Table 2$)$.
Similarly, the FasL indices of melanoma cells in Stage 3 and 4 melanoma cases were lower than those of Stage 1 and $2(P<.05$, each). As shown in Figure 3D-E, the Fas index of lymphocytes was 0.68 $\pm 0.09 \%$, and the FasL index was $1.05 \pm 0.18 \%$. There was no significant correlation between Fas indices of lymphocytes and melanoma stages (Table 3). Conversely, the FasL indices of lymphocytes from cases with Stage 3 and 4 disease were significantly lower than those from cases with Stage 1 and 2 disease.

Statistical analysis of the correlation between TUNEL and Fas or FasL indices revealed a highly positive correlation between the Fas and TUNEL indices of melanoma cells (Fig. 4A; $r=0.895, P<$ .0001). A positive correlation was also found between FasL and TUNEL indices of melanoma cells (Fig. 4B; $r=0.770, P<.001$ ), between Fas and TUNEL indices of lymphocytes (Fig. 5A; $r=0.747, P$ $<.05$ ), and between FasL and TUNEL indices of lymphocytes (Fig. 5B; $r=0.608, P<.01$ ). Moreover, the correlation between the TUNEL index of melanoma cells and the FasL index of lymphocytes was significantly positive (Fig. 6A; $r=0.729, P<.001$ ),

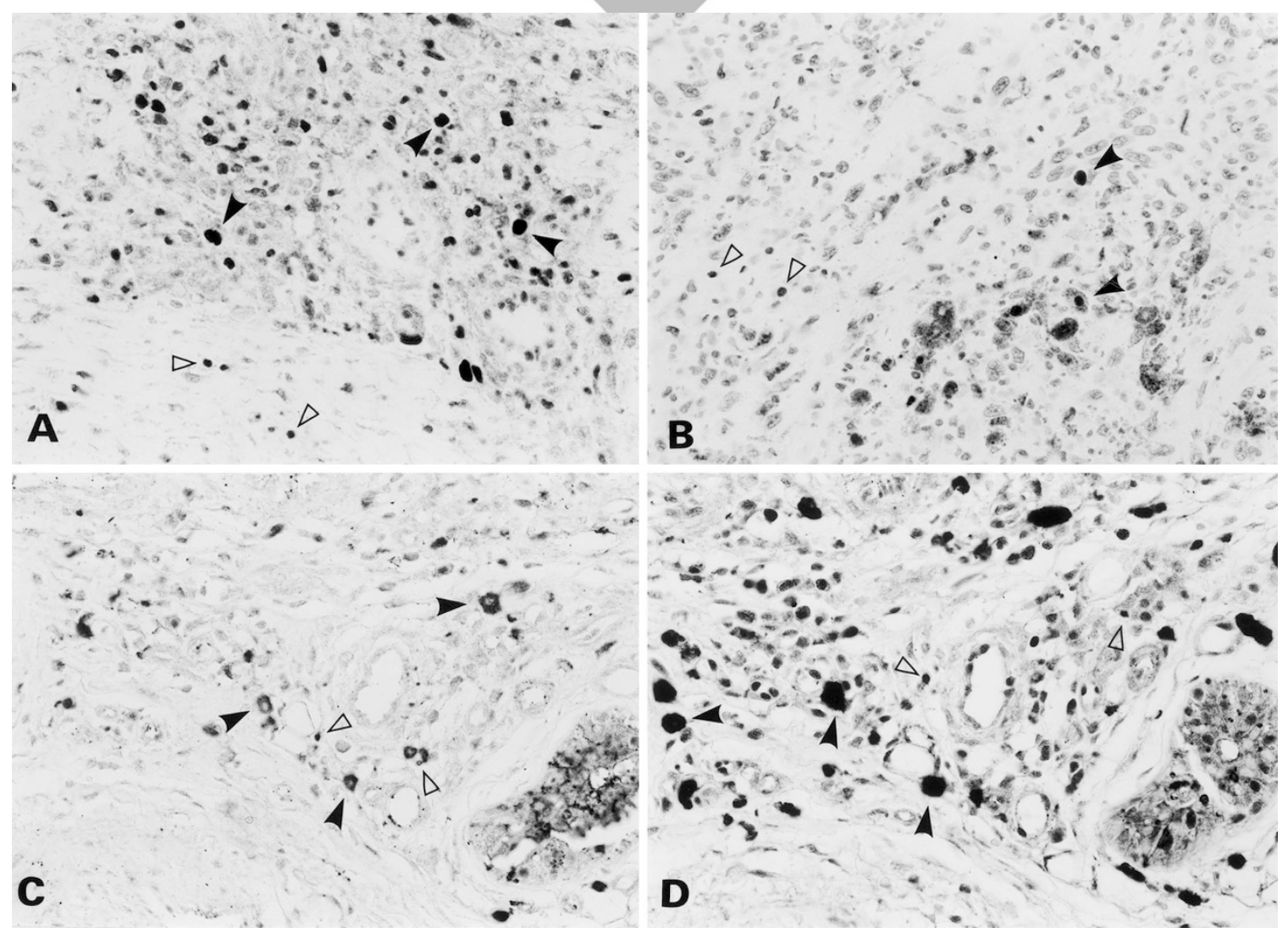

FIGURE 1. Staining for proliferating cell nuclear antigen (PCNA), DNA strand breaks (terminal deoxynucleotidyl transferase-mediated biotin-dUTP nick end-labeling; TUNEL), Fas, and FasL in melanoma tissue (Stage 2; Case 4). A, proliferating melanoma cells (closed arrowheads) and lymphocytes (open arrowheads) exhibited immunohistochemical staining for anti-PCNA antibody (230×). B, apoptotic melanoma cells (closed arrowheads) and lymphocytes (open arrowheads) were identified by TUNEL assay ( $230 \times)$. C, Fas-expressing melanoma cells (closed arrowheads) and lymphocytes (open arrowheads) were revealed by immunohistochemistry $(230 \times)$. D, in the serial section of Panel C, FasL-expressing melanoma cells (closed arrowheads) and lymphocytes (open arrowheads) were identified by immunohistochemistry (230 $\times$ ). 


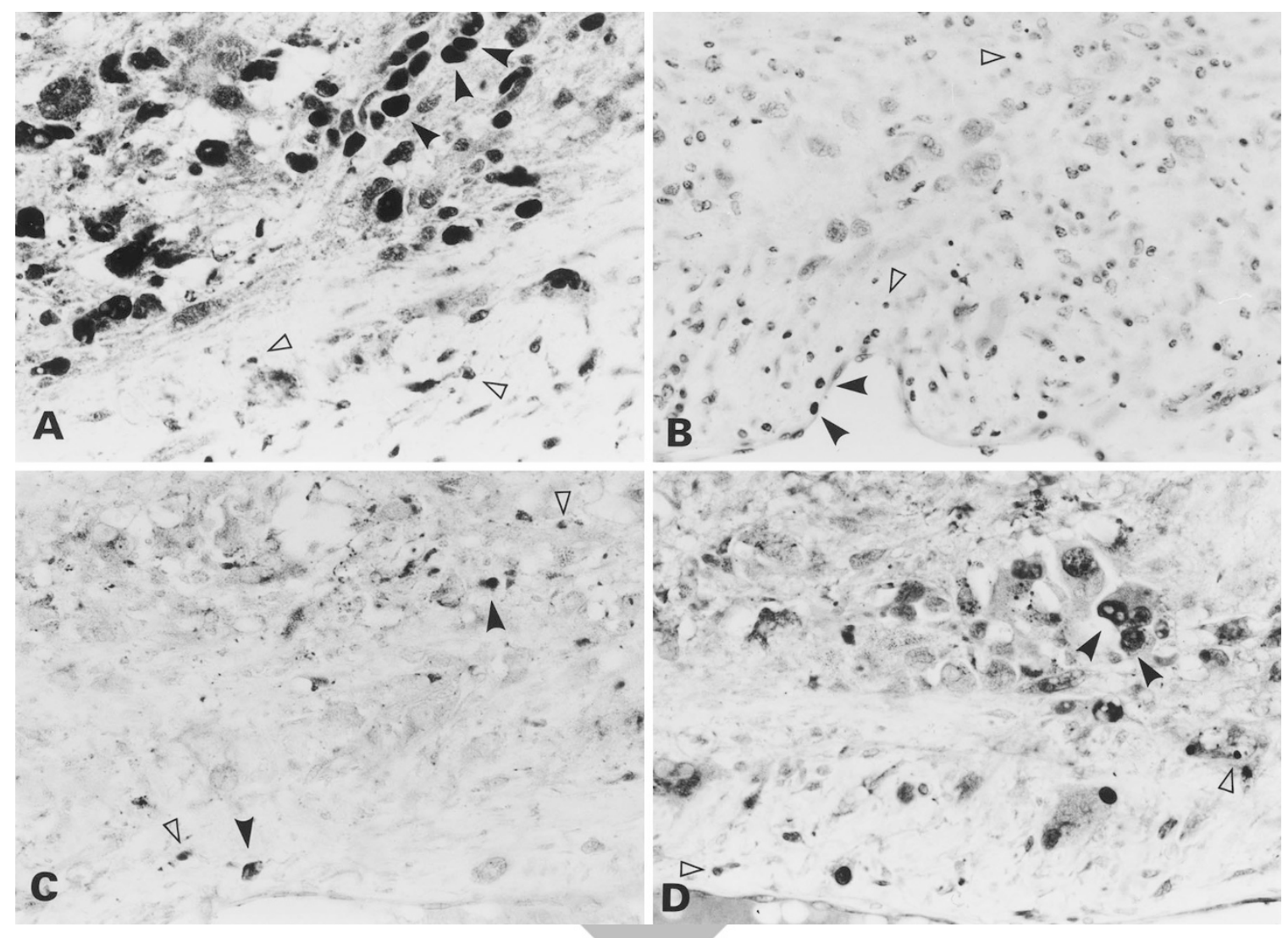

FIGURE 2. Staining for proliferating cell nuclear antigen (PCNA), DNA strand breaks (terminal deoxynucleotidyl transferase-mediated biotin-dUTP nick end-labeling; TUNEL), Fas, and FasL in melanoma tissue (Stage 4; Case 22). A, proliferating melanoma cells (closed arrowheads) and lymphocytes (open arrowheads) exhibited immunohistochemical staining for anti-PCNA antibody (230×). B, a few apoptotic melanoma cells (closed arrowheads) and lymphocytes (open arrowheads) were detected by TUNEL assay (230×). C, Fas-expressing melanoma cells (closed arrowheads) and lymphocytes (open arrowheads) revealed by immunohistochemistry $(230 \times)$. D, in the serial section of Panel C, a few FasL-expressing melanoma cells (closed arrowheads) and lymphocytes (open arrowheads) were identified by immunohistochemistry $(230 \times)$.

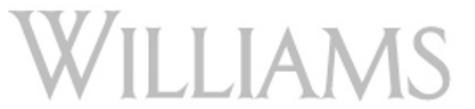

although the correlation between the TUNEL index of lymphocytes and FasL index of melanoma cells was marginal (Fig. 6B; $r=0.465, P<.05$ ).

\section{DISCUSSION}

In this study, we investigated the occurrence of apoptosis in malignant melanoma tissues and the possible involvement of the Fas and FasL system in apoptosis of melanoma cells and infiltrating lymphocytes. The major finding of our study was that Fas expression in melanoma cells correlated significantly with the frequency of apoptosis of melanoma cells and that apoptosis of melanoma cells affected the prognosis of melanoma patients. We also found that both melanoma cells and infiltrating lymphocytes can express Fas and FasL in clinical specimens, extending the earlier findings that Fas is expressed in infiltrating lymphocytes (21) and FasL is expressed in melanoma cells (21-24), though the lymphocyte apoptosis in itself was not significantly related to the prognosis. Therefore, these results indicate that the melanoma cell apoptosis mediated by the Fas-FasL system may affect

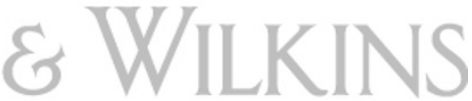

the growth of melanoma and the prognosis of melanoma patients.

Because the growth of malignant tumors depends on imbalance between cell proliferation and cell loss (1), knowing only the proliferation rate or only the apoptotic rate is not sufficient to understand the kinetics of tumor growth. The correlation between proliferation and apoptosis has recently been investigated in malignant epidermal neoplasms, including squamous cell carcinoma, basal cell carcinoma, and Bowen's disease (30). Although malignant melanoma is associated with a poor prognosis compared with these epidermal neoplasms, only a few reports have emerged concerning the relationship between proliferation and apoptosis of malignant melanoma cells (13). In the present study, we first assessed the TUNEL-PCNA ratio of melanoma cells as a tentative parameter of the balance between cell proliferation and cell death and found that the TUNEL-PCNA ratio was inversely correlated with melanoma stage. Considering that the PCNA index or TUNEL index of melanoma cells alone was not sufficient to discriminate Stages 1 to 3, we suggest that the TUNEL-PCNA 

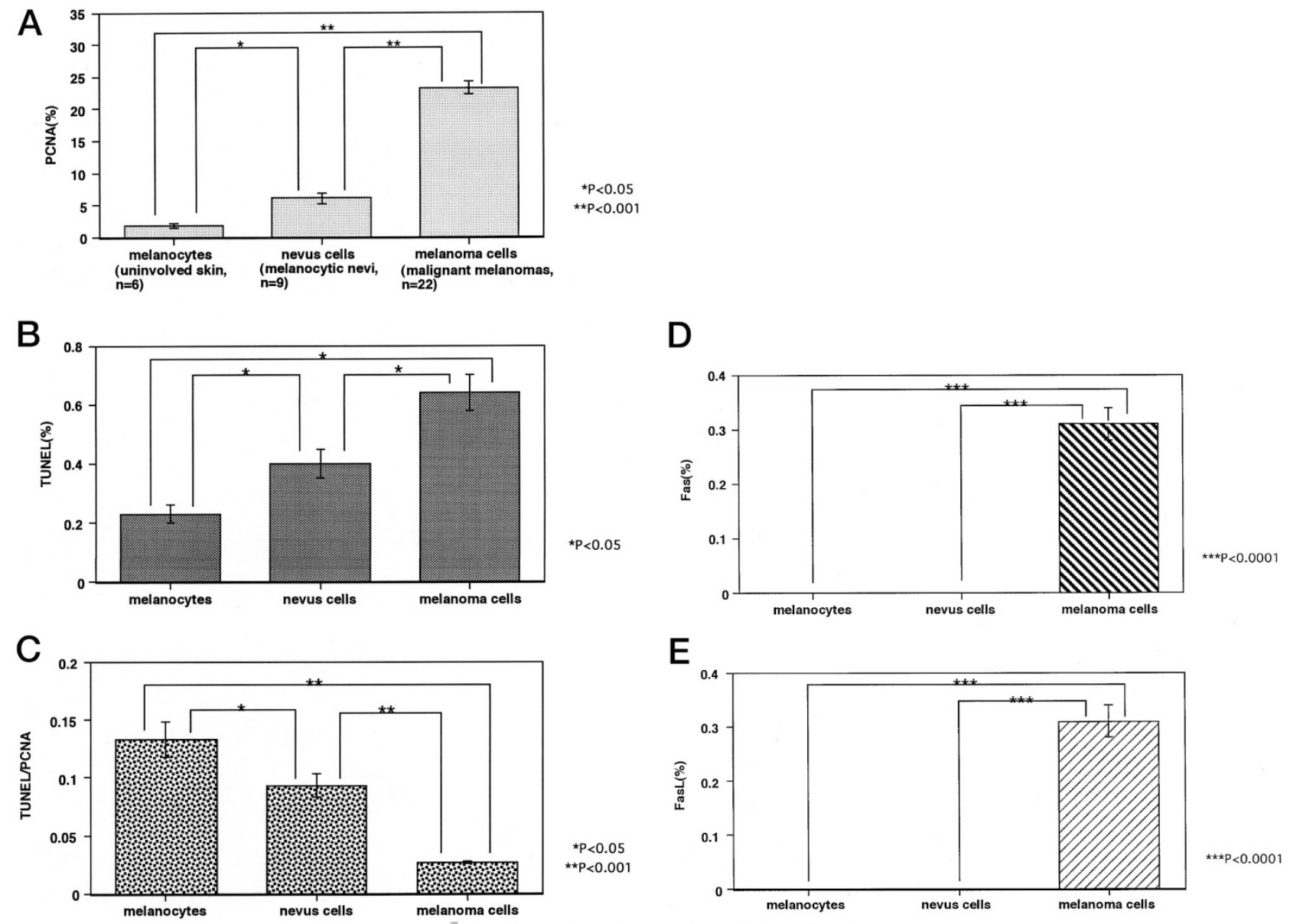

FIGURE 3. Various indices of melanoma cells, nevus cells, and melanocytes. A, proliferating cell nuclear antigen (PCNA) index, B, terminal deoxynucleotidyl transferase-mediated biotin-dUTP nick end-labeling (TUNEL) index, C, TUNEL-PCNA ratio, D, Fas index, and E, FasL index. Values are means \pm SEM. The indices of lymphocytes in melanoma tissue were $2.10 \pm 0.27 \%$ in PCNA, $3.82 \pm 0.58 \%$ in TUNEL, $2.162 \pm 0.270$ in TUNEL-PCNA, $0.68 \pm 0.09 \%$ in Fas, and $1.05 \mp 0.18 \%$ in FasL.

TABLE 2. PCNA, TUNEL, Fas and FasL Indices of Melanoma Cells from Stage 1 to 4

\begin{tabular}{|c|c|c|c|c|c|}
\hline $\begin{array}{c}\text { Melanoma } \\
\text { Cells }\end{array}$ & PCNA (\%) & TUNEL (\%) & TUNEL/PCNA & Fas (\%) & FasL (\%) \\
\hline Stage $1(\mathrm{n}=4)$ & $15.98 \pm 2.41$ & $0.80 \pm 0.10$ & $0.051 \pm 0.002$ & $0.37 \pm 0.05$ & $0.41 \pm 0.07$ \\
\hline Stage $2(n=5)$ & $24.76 \pm 0.42$ & $0.90 \pm 0.12$ & $0.037 \pm 0.005$ & $0.44 \pm 0.03$ & $0.61 \pm 0.02$ \\
\hline Stage $3(n=6)$ & $25.54 \pm 0.96$ & $0.60 \pm 0.11$ & $0.023 \pm 0.004$ & $0.29 \pm 0.05$ & $0.26 \pm 0.09$ \\
\hline Stage $4(\mathrm{n}=7)$ & $24.85 \pm 1.09$ & $0.39 \pm 0.08$ & $0.016 \pm 0.003$ & $0.30 \pm 0.06$ & $0.16 \pm 0.04$ \\
\hline
\end{tabular}

$* p<0.05$

TABLE 3. PCNA, TUNEL, Fas and FasL Indices of Lymphocytes from Stage 1 to 4

\begin{tabular}{cccr}
\hline Lymphocytes & PCNA (\%) & TUNEL (\%) & Fas (\%) \\
\hline Stage 1 $(\mathrm{n}=4)$ & $1.45 \pm 0.39$ & $4.59 \pm 1.71$ & $0.76 \pm 0.26$ \\
Stage 2 $(\mathrm{n}=5)$ & $2.69 \pm 0.61$ & $6.24 \pm 1.99$ & $1.06 \pm 0.18$ \\
Stage 3 $(\mathrm{n}=6)$ & $2.32 \pm 0.45$ & $4.07 \pm 1.23$ & $0.68 \pm 0.20$ \\
Stage 4 $(\mathrm{n}=7)$ & $1.96 \pm 0.60$ & $3.16 \pm 1.05$ & 0.55 \\
\hline
\end{tabular}

$* p<0.05$.

ratio may be a better parameter to predict the prognosis of malignant melanoma patients.

Maeda et al. (22) have recently shown that nevus cells are FasL negative. In accordance with this finding, we also found benign nevus cells and melanocytes to be negative for FasL and that both cell types were negative for Fas. Conversely, Fas and FasL indices of melanoma cells were greater than those of nevus cells and melanocytes. Moreover, the TUNEL and Fas indices and the TUNEL-PCNA ratio of melanoma cells in Stage 3 melanomas were significantly lower than those in Stage 2 melanomas. In Stage 3, the thickness of primary tumor is $>4.0 \mathrm{~mm}$, or local regional lymph node involvement is present. In Stages 1 and 2, there is no metastatic lesion. Therefore, it might be possible 
A

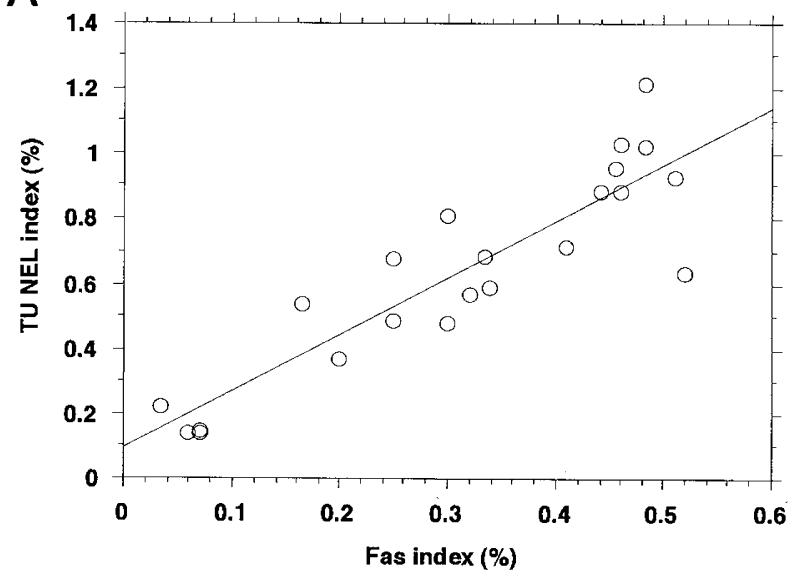

B

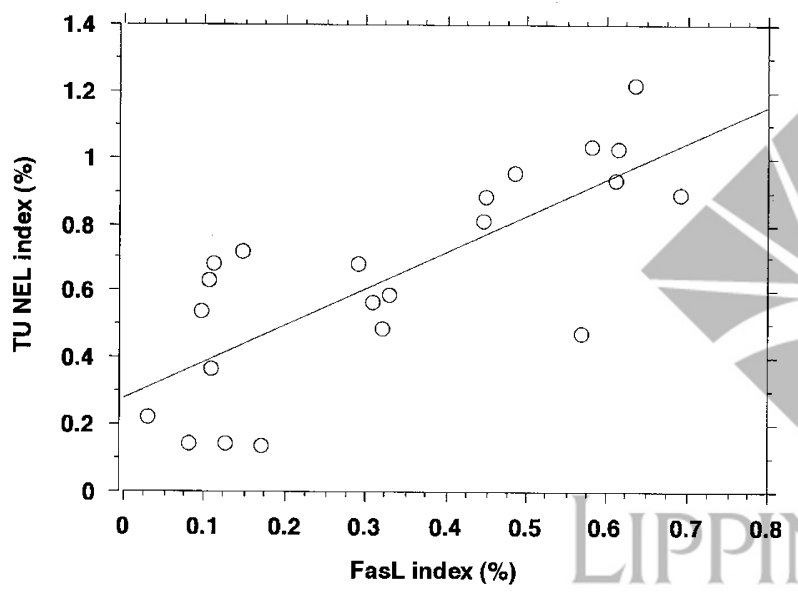

FIGURE 4. A, correlation between Fas index and terminal deoxynucleotidyl transferase-mediated biotin-dUTP nick end-labeling (TUNEL) index of melanoma cells. $r \neq 0.895, P<.0001$, Spearman's rank correlation test. B, correlation between FasL index and TUNEL index of melanoma cells. $r=0.770, P<.001$, Spearman's rank correlation test.

that the changes in the expression of Fas and FasL genes, accompanying the induction of melanoma cell apoptosis, are linked with any genetic disorders that follow the acquisition of the metastatic activity by melanoma cells. On the other hand, the difference in TUNEL, Fas, and FasL indices of melanoma cells was small between Stages 1 and 2, although the PCNA index of Stage 2 was significantly greater than that of Stage 1 . Stage 1 is the early stage of melanoma, and the progression of melanomas from Stage 1 to Stage 2 may be mainly associated with the acceleration of proliferating activity in melanoma cells. In invasive stages, including Stages 3 and 4, the decline of apoptosis of melanoma cells would be more important to prognosis of melanoma patients than would the proliferation of those cells. Furthermore, the difference in PCNA, TUNEL, Fas, and FasL indices of melanoma cells was small between Stages 3 and 4, indicating that the stages of features might be an extension of the Stage 3 mel-
A

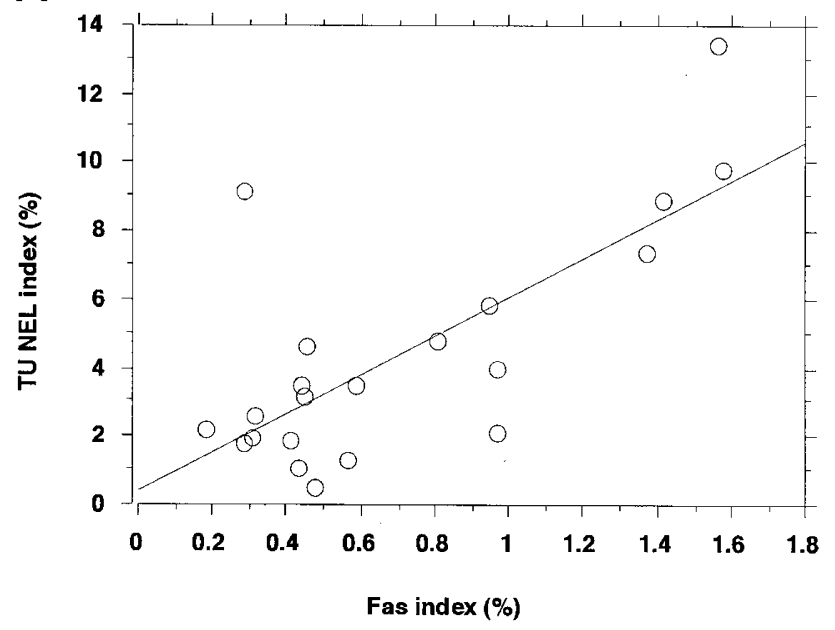

B

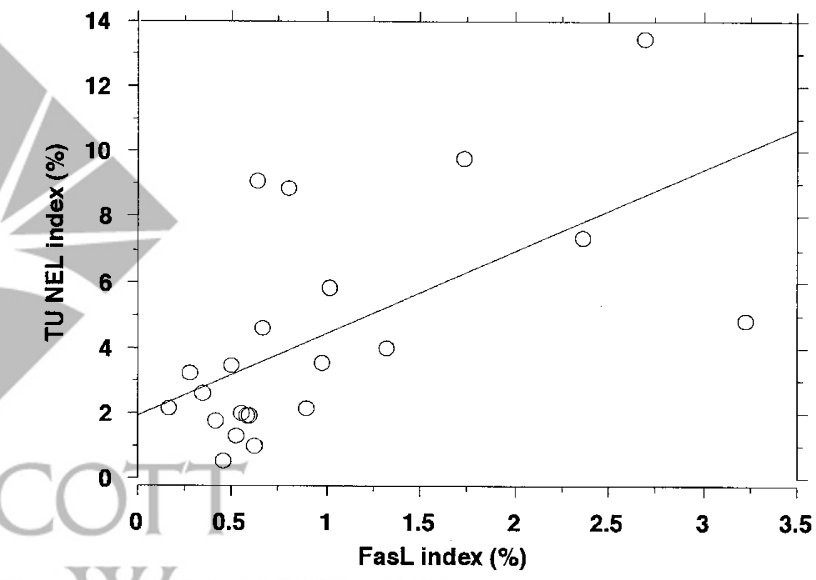

FIGURE 5. A, correlation between Fas index and terminal deoxynucleotidyl transferase-mediated biotin-dUTP nick end-labeling (TUNEL) index of lymphocytes. $r=0.747, P<.05$, Spearman's rank correlation test. B, correlation between FasL index and TUNEL index of lymphocytes. $r=0.608, P<.01$, Spearman's rank correlation test. anoma without any further alterations in proliferation or apoptotic activity. Thus, the low frequency of apoptosis and Fas expression may contribute to the poor prognosis of malignant melanoma. These findings indicate that a critical point for molecular therapy for malignant melanoma may be the increase of Fas expression in melanoma cells.

Statistical analysis of the correlation between TUNEL and Fas or FasL indices revealed that the most positive correlation was between the TUNEL and Fas indices of melanoma cells. Recently, it has been also reported that melanoma cells that were induced to express Fas by transfection of the Fas gene underwent apoptotic cell death in vivo (31). These results strongly indicate that Fas expression may be tightly associated with induction of apoptosis in melanoma cells. However, in the present study, we did not observe direct evidence that Faspositive cells were also TUNEL positive in serial sections. In this context, it should be noted that 

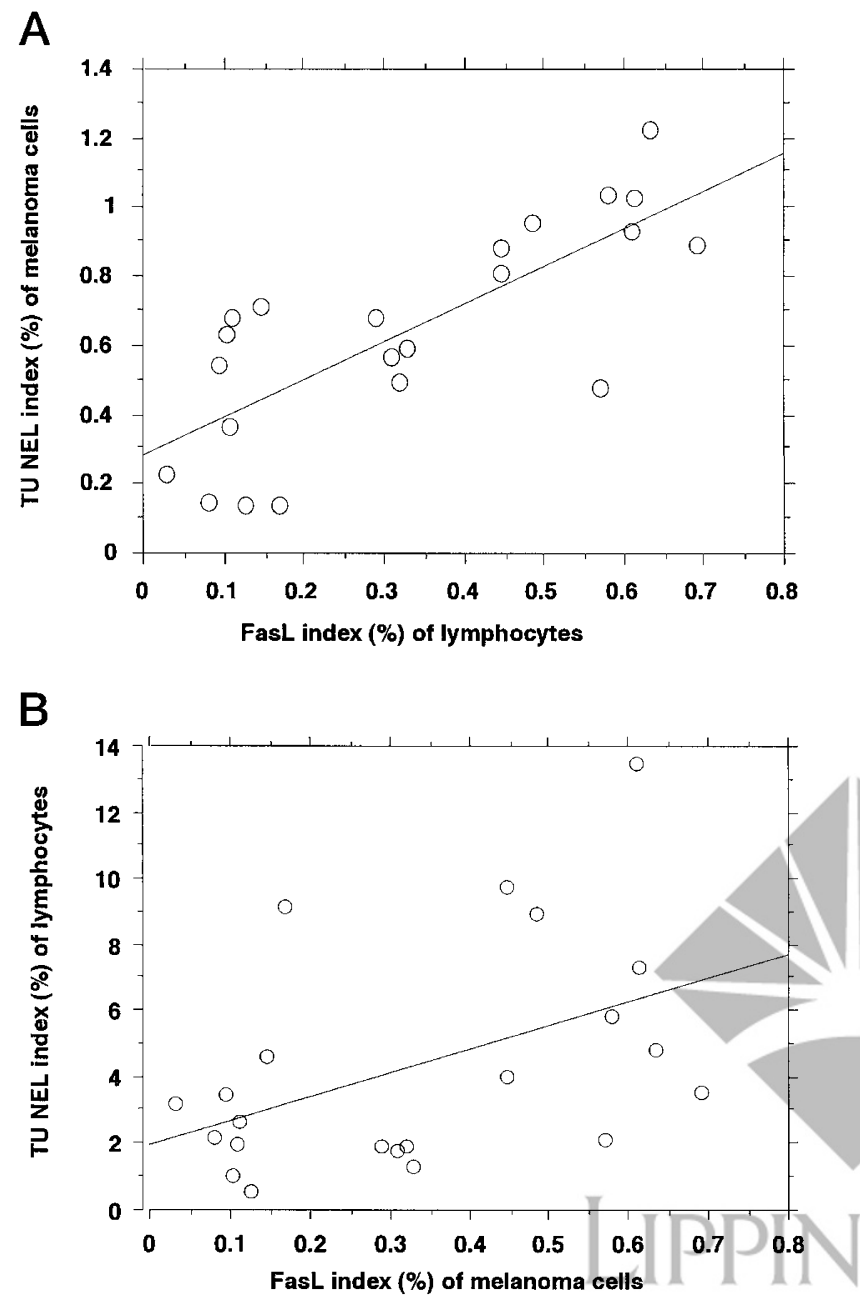

FIGURE 6. A, correlation between FasL index of lymphocytes and terminal deoxynucleotidyl transferase-mediated biotin-dUTP nick endlabeling (TUNEL) index of melanoma cells. $r=0.729, P<.001$,

Spearman's rank correlation test. B, correlation between FasL index of melanoma cells and TUNEL index of lymphocytes. $r=0.465, P<.05$, Spearman's rank correlation test.

\section{Unaut tho} apoptotic cells may no longer express Fas antigen
because dying cells would not alter their gene expression profile (18). Furthermore, it is not clear whether cell surface antigens are maintained during the apoptotic process.

In accordance with our findings, recent flowcytometric analysis has revealed that Fas-positive melanoma cells decreased as primary melanoma thickness progressively increased (32). In addition, it has been reported that the increased soluble Fas level correlates with poor prognosis in advanced melanomas $(33,34)$ and that the soluble FasL levels in melanoma patients are higher than those in healthy donors (34). Because soluble Fas binds FasL on cell surface or soluble FasL, the increase of soluble Fas may result in the protection of melanoma cells from the Fas-FasL-dependent apoptosis. In this content, it should be noted that Fas and FasL indices of melanoma cells and lymphocytes de- creased in Stage 3 and 4 melanomas. So, it is possible that soluble Fas and FasL come out from the surface of melanoma cells and lymphocytes in these advanced stages of melanoma.

Recently, investigation of apoptosis of Faspositive lymphocytes induced by FasL-expressing tumor cells has been focused on tumor escape from immunological rejection in various malignant neoplasms, including hepatocellular carcinoma (7), colon cancer $(18,35)$, and melanoma $(21,23,24)$. Hahne (21) reported that FasL-expressing melanoma cells and Fas-positive lymphocytes were found in metastatic melanoma lesions. In our present study, we also detected Fas-expressing melanoma cells and FasL-expressing lymphocytes, together with Fas-expressing lymphocytes and FasLexpressing melanoma cells in the primary lesions. Thus, it is possible that apoptosis of Fas-positive melanoma cells may be induced by FasL-expressing lymphocytes and melanoma cells. With respect to the apoptosis of Fas-positive lymphocytes, it is possible that apoptosis of Fas-positive lymphocytes might be induced by FasL-expressing melanoma cells and lymphocytes in any stage. Indeed, we confirmed a significant correlation between the TUNEL index of lymphocytes and the FasL index of melanoma cells. However, the PCNA, TUNEL, and TUNEL-PCNA indices of lymphocytes did not correlate with melanoma stage. Furthermore, the indices of PCNA, TUNEL, Fas, and FasL were not significantly different among stages in our study. Thus, it might be possible that the apoptosis of Tymphocytes would be less important for progression of melanomas than that of melanoma cells. Rather, the apoptosis of melanoma cells attacked by lymphocytes and melanoma cells themselves should be necessary for therapy.

In conclusion, our study showed that melanoma cells and infiltrating lymphocytes may undergo apoptosis mediated through Fas and FasL in an autocrine and/or paracrine manner and that Fasmediated apoptosis of melanoma cells may affect the prognosis of melanoma patients.

Acknowledgments: The authors thank Shinichi Izumi, Masashi Shin, Kuniko Abe, Yoshitaka Hishikawa (Department of Histology and Cell Biology, Nagasaki University School of Medicine), and Teruhide Masuda (Department of Dermatology, Nagasaki University School of Medicine) for their helpful advice and excellent technical support.

\section{REFERENCES}

1. Cotran RS. Neoplasia. In Cotran RS, Kumar V, Robins SL, editors. Robins' pathologic basis of the disease, 4th ed. Philadelphia: Saunders; 1989. p. 239-306. 
2. Hakuno N, Koji T, Yano T, Kobayashi M, Tsutsumi O, Taketani Y, et al. Fas/APO-1/CD95 system as a mediator of granulosa cell apoptosis in ovarian follicle atresia. Endocrinology 1996;137:1938-48.

3. Wang R, Nakane PK, Koji T. Autonomous cell death of mouse male germ cells during fetal and postnatal period. Biol Reprod 1998;58:1250-6.

4. Tamura M, Kimura H, Koji T, Tominaga T, Ashizawa K, Kiriyama $\mathrm{T}$, et al. Role of apoptosis of thyrocytes in a rat model of goiter. A possible involvement of Fas system. Endocrinology 1998;139:3646-53.

5. Kusiak JW, Izzao JA, Zhao B. Neurodegeneration in Alzheimer disease. Is apoptosis involved? Mol Chem Neuropathol 1996;28:152-62.

6. Cohen JJ, Izzao JA, Zhao B. Programmed cell death in the immune system. Adv Immunol 1991;50:55-85.

7. Rubio CA. Tumor cells induce apoptosis in lymphocytes. Nat Med 1997;3:253-4.

8. Jaattela M, Benedict M, Tewari M, Shayman JA, Dixit VM. Bcl-X and Bcl-2 inhibit TNF, and Fas-induced apoptosis and activation of phospholipase A2 in breast carcinoma cells Oncogene 1995;10:2297-305.

9. Tamura G, Kihara T, Nomura K, Terada M, Sugimura T, Hirohashi S. Detection of frequent p53 gene mutations in primary gastric cancer by cell sorting and polymerase chain reaction single-strand conformation polymorphism analysis. Cancer Res 1991;51:3056-8.

10. Ionov Y, Peinado M, Malkhosyan S, Shibata D, Perucho M. Ubiquitous somatic mutations in simple repeated sequences reveal a new mechanism for colonic carcinogenesis. Nature 1993;363:558-61.

11. Nakagawa S, Shiraishi T, Kihara S, Tabuchi K. Detection of DNA strand breaks associated with apoptosis in human brain tumors. Virchows Res 1995;427:175-9.

12. Boring CC, Squires TS, Tong T, Montgomery S. Cancer statistics 1994. CA Cancer J Clinic 1994;44:7-26.

13. Mooney E, Peris J, O'Neill A, Sweeney E. Apoptotic and mitotic indices in malignant melanoma and basal cell carcinoma. J Clin Pathol 1995;48:242-4.

14. Saida T, Dohi S, Takasaki Y. Distribution patterns and frequency of proliferating cells in cutaneous keratinocytic neoplasm. J Am Acad Dermatol 1992;26:744-8.

15. Kerr JFR, Wyllie AH, Currie AR. Apoptosis. A basic biological phenomenon with wide-ranging implications in tissue kinetics. Br J Cancer 1972;26:239-57.

16. Gavrieli Y, Sherman Y, Ben-Sasson SA. Identification of programmed cell death in situ via specific labeling of nuclear DNA fragmentation. J Cell Biol 1992;119:493-501.

17. Yonehara S, Ishii A, Yonehara M. A cell-killing monoclonal antibody (anti-Fas) to cell surface antigen co-downregulated with the receptor of the tumor necrosis factor. J Exp Med 1989;169:1747-56.

18. Okada K, Komuta K, Hashimoto S, Matsuzaki S, Kanematsu T, Koji T. Frequency of apoptosis of tumor-infiltrating lymphocytes induced by Fas counterattack in human colorectal carcinoma and its correlation with prognosis. Clin Cancer Res 2000;6:3560-4.

19. Suda T, Nagata S. Purification and characterization of the Fas-ligand that induces apoptosis. J Exp Med 1994;179:873879.
20. Dhein J, Walczak H, Baumler C, Debatin KM, Krammer PH. Autocrine T-cell suicide mediated by APO-1/(Fas/CD95). Nature 1995;373:438-41.

21. Hahne M, Rimoldi D, Schràter M, Romero P, Schreier M, French LE, et al. Melanoma cell expression of Fas(Apo-1/ CD95) ligand: implications for tumor immune escape. Science 1996;274:1363-6.

22. Maeda A, Aragane Y, Tezuka T. Expression of CD95 ligand in melanocytic lesions as a prognostic marker. Br J Dermatol 1998;139:198-206.

23. Sprecher E, Bergman R, Meilick A, Kerner H, Manov L, Reiter I, et al. Apoptosis, Fas and Fas-ligand expression in melanocytic tumors. J Cutan Pathol 1999;26:72-7.

24. Terhyden P, Siedel C, Merkel A, Kampgen E, Brocker EB, Becker JC. Predominant expression of Fas (CD95) ligand in metastatic melanoma revealed by longitudinal analysis. J Invest Dermatol 1999;112:899-902.

25. Koji T, Kobayashi N, Nakanishi Y, Yoshii A, Hashimoto S, Shibata Y, et al. Immunohistochemical localization of Fas antigen in paraffin sections with rabbit antibodies against human synthetic Fas peptides. Acta Histochem Cytochem 1994;27:459-63.

26. Suda T, Takahashi T, Golstein P, Nagata S. Molecular cloning and expression of the Fas ligand, a novel member of the tumor necrosis factor family. Cell 1993;75:1169-78.

27. Clark WH Jr, From L, Bernardino EA, Mihm MC. The histogenesis and biological behavior of primary human malignant melanomas of the skin. Cancer Res 1969;29: 705-27.

28. Kuchelmeister C, Schaumburg-Lever G, Garbe C. Acral cutaneous melanoma in Caucasians: clinical features, histopathology and prognosis in 112 patients. Br J Dermatol 2000; 143:275-80.

29. Adams JC. Heavy metal intensification of DAB-based HRP reaction product. J Histochem Cytochem 1981;29:775.

30. Makino T, Tatebe S, Goto A, Mihara M, Ito H. Apoptosis and cellular proliferation in human epidermal squamous cell neoplasia. J Cutan Pathol 1998;25:136-42.

31. Aragane Y, Maeda A, Cui CY, Tezuka T, Kameda Y, Schwarz $\mathrm{T}$. Inhibition of growth of melanoma cells by CD95 (Fas/ Apo-1) gene transfer in vivo. J Invest Dermatol 2000;115: 1008-14.

32. Soubrane C, Mouawad R, Antoine EC, Verola O, Gil-Delgado M, Khayat D. A comparative study of Fas and Fas-ligand - expression during melanoma progression. Br J Dermatol 2000;143:307-12.

33.=Ugurel S, Rappl G, Tilgen W, Reinhold U. Increased soluble CD95 (sFas/CD95) serum level correlates with poor prognosis in melanoma patients. Clin Cancer Res 2001;7: 1282-6.

34. Mouawad R, Khayat D, Soubrane C. Plasma Fas ligand, an inducer of apoptosis, and plasma soluble Fas, an inhibitor of apoptosis, in advanced melanoma. Melanoma Res 2000;10: 461-7.

35. O'Connel J, O'Sullivan GC, Collins JK, Shanahan F. The Fas counterattack. Fas-mediated T cell killing by colon cancer cells expressing Fas ligand. J Exp Med 1996;184: 1075-82. 